\title{
On the estimation of misclassification probabilities of chronic kidney disease using continuous time hidden Markov models
}

\author{
Gurprit Grover $^{1}$, Alka Sabharwal' ${ }^{2}$, Shrawan Kumar ${ }^{*} \mathbb{D}$, Arpan Kumar Thakur $^{1}$ \\ ${ }^{1}$ Department of Statistics, University of Delhi. Delhi, India \\ ${ }^{2}$ Department of Statistics, Kirori Mal College, University of Delhi, Delhi, India
}

\section{A R T I C L E I N F O}

Article Type:

Original

\section{Article History:}

Received: 8 July 2018

Accepted: 8 October 2018

ePublished: 10 November 2018

\section{Keywords:}

Chronic kidney disease

Hidden Markov model

Glomerular filtration rate

Transition intensity

Mean sojourn time

Viterbi algorithm

\begin{abstract}
A B S T R A C T
Introduction: The severity of chronic kidney disease (CKD) is reflected in the form of stages of CKD and can be decided on the basis of estimated glomerular filtration rate (eGFR). The computation of eFGR may have computational and measurement errors which may lead to misclassification of stages.

Objectives: Estimation of transition rates, mean sojourn times, probabilities of misclassification of stages and odds ratios for misclassification probabilities.

Patients and Methods: The retrospective data of 117 patients suffering from CKD during the period March 2006 to October 2016 is used. Hidden Markov model (HMM) with continuous time has been developed to present the course of progression of CKD into various stages.

Results: Under the HMM, the estimated transition intensity corresponding to transition from stage 1 to stage 2 is 0.0405 and reverse transition intensities are zero. The estimated mean sojourn time corresponding to stage 1, stage 2, stage 3 and stage 4 are 15.923 years, 11.976 years, 7.936 years and 2.890 years respectively. The probability of a CKD patient with stage 1 of disease will be misclassified as a patient of stage 2 is 0.211 . The odds ratios for misclassification probabilities in the presence of prognostic factors are computed. The probability of misclassification corresponding to the observed stage 2 given the true stage 1 for females is approximately 3.8 times more than that of males.

Conclusion: For CKD having progression in stages, the HMM is an appropriate model to draw the course of progression of the disease.
\end{abstract}

\section{Implication for health policy/practice/research/medical education:}

$\mathrm{CKD}$ is asymptomatic in its early stages and the determination of stage may be erroneous due to other prognostic factors which may lead to misclassification of stages. Medical practitioners should consider all the factors while screening a patient before declaring the stage of the disease.

Please cite this paper as: Grover G, Sabharwal A, Kumar S, Thakur AK. On the estimation of misclassification probabilities of chronic kidney disease using continuous time hidden Markov models. J Nephropharmacol. 2019;8(1):e07. DOI: 10.15171/ npj.2019.07

\section{Introduction}

Chronic kidney disease (CKD) is defined as the presence of kidney damage along with the reduction in the level of kidney function. According to National Kidney Foundation (NKF) recommendations, and based on the estimated glomerular filtration rate (eGFR) classification intervals, $\mathrm{CKD}$ can be classified into five categories of disease stages. The worldwide prevalence of CKD is varying between $10.5 \%$ and $13.1 \%$ (1). In Western countries, $2 / 3^{\text {rd }}$ cases of CKD are due to diabetes and hypertension (2). The prevalence of CKD in India is approximately 800 per million populations (3).

The progression of $\mathrm{CKD}$ can be shown by Markov process permitting only the forward transitions from one state to another state over time (4). The progression of the disease is continuous in time and the time of transitions are random in nature. Homogeneous continuous time multistate model based on Markov processes is the suitable model to describe the course of progression of CKD (5). In the case of hidden Markov model (HMM), it is assumed that the true stages of disease are hidden (unobservable or latent). The actual stage of disease can only be determined indirectly with the help of the disease marker. It is assumed that the generated observations are conditionally independent given the true stages. HMM has been extensively applied in areas of speech and signal 
processing (6). Chen et al proposed anHMM for breast cancer screening (7). Kirby et al described HMM in the case of cervical smear tests (8), Satten and Longini (9) and Guihenneuc-Jouyaux et al (10) used HMM for describing the progression of HIV infection to different stages on the basis of CD 4 cell counts. Jackson et al used HMM model for representing the decline in the function of the lung after lung transplantation (11).

In the current study, homogeneous continuous-time HMM has been used to study the progression of CKD. We have estimated the transition rates between various states and probabilities of misclassification between the true and observed stages of the disease. Using these parameters, sojourn times of states and conditional probabilities are also computed. The mean sojourn time of a state is the length of time a CKD patient spends on an average in that stage before moving to the next stage (characterized by a state of the Markov chain).

We have also investigated the impact of prognostic factors such as diabetes, hypertension and age on transition rates and misclassification probabilities. The odds ratios of misclassification probabilities have been computed in the presence of covariates (prognostic factors). To the best of our knowledge, HMM has not been applied to study the course of progression of CKD. Section 1 includes introduction and objective of the study. Section 2 deals with the materials and methods, results are presented in section 3 followed by discussions and conclusions in section 4 and section 5 respectively.

\section{Objectives}

This study aimed to ascertain whether the observed stages based on eGFR match with the true stages of CKD. In addition, we aimed to determine the most likely misclassified stage using an appropriate model and its impact on the progression of the disease.

\section{Patients and Methods \\ Study population}

For the present study the retrospective data of 117 patients suffering from CKD during the period March 2006 to October 2016 is used. The registered laboratories and hospitals were approached for the records of CKD patients. We contacted 550 patients, however more than 50\% were reluctant to share their data. Only 248 patients responded positively. Informed consent was taken from all of them. After scrutiny, data of 117 patients were found to be suitable for the present study. The information such as gender, age, diabetes, hypertension, body mass index, hemoglobin level, urea, serum creatinine and albumin were recorded for each patient. The data are the observed stages of CKD based on the value of eGFR with individual-specific values of covariates. The five stages of chronic disease based on the value of GFR are; stage $1\left(\mathrm{GFR} \geq 90 \mathrm{ml} / \mathrm{min} / 1.73 \mathrm{~m}^{2}\right)$; stage $2\left(60 \leq \mathrm{GFR} \leq 89 \mathrm{~mL} / \mathrm{min} / 1.73 \mathrm{~m}^{2}\right)$; stage $3(30 \leq$ GFR $\left.\leq 59 \mathrm{~mL} / \mathrm{min} / 1.73 \mathrm{~m}^{2}\right)$; stage $4(15 \leq \mathrm{GFR} \leq 29 \mathrm{~mL} /$ $\left.\mathrm{min} / 1.73 \mathrm{~m}^{2}\right)$ and stage $5\left(\mathrm{GFR}<15 \mathrm{~mL} / \mathrm{min} / 1.73 \mathrm{~m}^{2}\right)$. Stages 1, 2, 3 and 4 are transient states that is movements from these states to other states in the forward direction are allowed. Stage 5 ( ESRD) is an absorbing state and the movement from this state to any other state is not possible. It shows the loss of kidney functions and requires either dialysis or kidney transplantation. The number of followup time points for each patient was different and visiting times were irregular. The latest recorded stage of each patient is corresponding to the last follow-up time of the patient. The covariates considered are gender ( 0 female, 1 male), age, diabetes ( 0 No, 1 Yes) hypertension (0 No, 1 Yes), body mass index, hemoglobin level, urea, serum creatinine and albumin.

Homogeneous continuous-time multistate Markov model A homogeneous continuous-time multistate Markov model is an appropriate model for describing the progression of CKD. In this model, the observed states are precisely the same as true states of disease. States of Markov process are calculated on the basis of eGFR values. Observation times are taken as noninformative (12). A patient may make forward transition only among different transient states continuously. The arrows show the possible transition between stages.

The progression of CKD is presented below in Figure 1. Where the intensity $\lambda_{k l}$ is the instantaneous risk of moving from state $k$ to $l$.

$$
\lambda_{k l}=\lim _{\delta t \rightarrow 0} \frac{P(S(t+\delta t)=l \mid S(t)=k)}{\delta t}
$$

The state transition matrix $Q$ associated with Figure 1 is given by

$$
Q=\left(\begin{array}{ccccc}
-\left(\lambda_{12}+\lambda_{13}+\lambda_{14}+\lambda_{15}\right) & \lambda_{12} & \lambda_{13} & \lambda_{14} & \lambda_{15} \\
0 & -\left(\lambda_{23}+\lambda_{24}+\lambda_{25}\right) & \lambda_{23} & \lambda_{24} & \lambda_{23} \\
0 & 0 & -\left(\lambda_{34}+\lambda_{35}\right) & \lambda_{34} & \lambda_{35} \\
0 & 0 & 0 & -\lambda_{45} & \lambda_{45} \\
0 & 0 & 0 & 0 & 0
\end{array}\right)
$$

Sum of the entries in each row of transition matrix is zero. The diagonal element of $\mathrm{Q}$ is $\lambda_{k k}=-\sum_{k \neq l} \lambda_{k l}$.

Let $\mathrm{P}(\mathrm{t})$ denotes the transition probability matrix with transition probabilities $\mathrm{p}_{k l}(\mathrm{t}), k=1,2, \ldots, 5$ and $l=1,2, \ldots, 5$ as its elements,

Where, $\mathrm{p}_{k l}(t)=\operatorname{Pr}\left\{S_{i}(t+u)=l \mid S_{i}(t)=k\right\}$.

The likelihood function of transition intensities is the product of probabilities of transition between observed states over all individuals and observation times. The likelihood $\mathrm{L}(\mathrm{Q})$ is maximized in term of $\log \left(\lambda_{k l}\right)$. Therefore; the estimates $\lambda_{k l}$ are obtained from $\log \left(\lambda_{k l}\right)$ using optimization technique.

The maximum likelihood estimates of the transition 


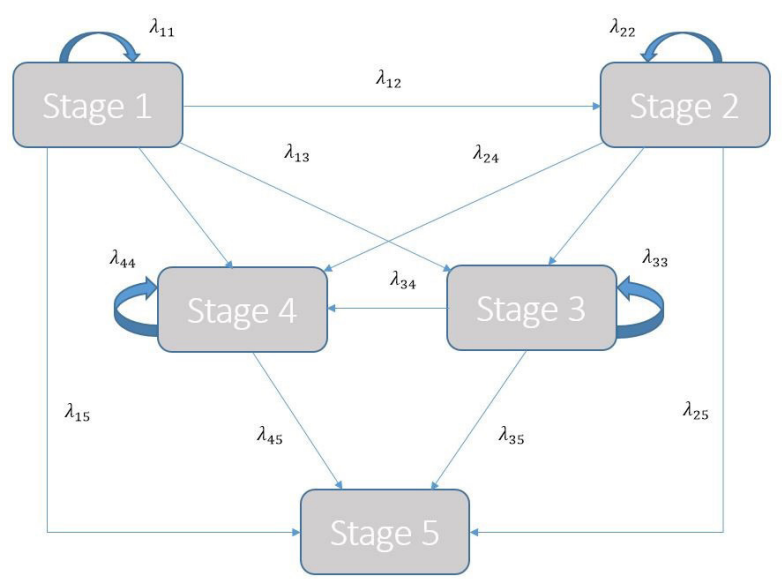

Figure 1. State transition diagram.

intensities have been obtained which in turn enable us to obtain $P(t)=\exp (t Q)(13)$.

Mean sojourn time for states can be computed from the estimated transition rates as $\frac{-1}{\lambda_{k k}}$. The impact of explanatory variables (prognostic factors) on particular transition intensity can be explained by modeling the transition intensity as a function of these covariates which results in transition probability matrix $P(t, x(t))$. In such case, the new transition matrix $Q$ is used in the likelihood function for estimating the transition intensities. Marshall and Jones used the proportional hazards model for studying the effect of a vector of explanatory variables on transition intensity for the individual $i$ at a time $j$ by replacing the transition intensity element $\lambda_{k l}$ by $\lambda_{k l}\left(z_{i j}\right)=\lambda_{k l}^{(0)} \exp \left(\beta_{k l}^{T} \mathrm{z}_{i j}\right)$. The maximum likelihood estimates for baseline intensities (14) and regression coefficients are obtained using the msm package in $\mathrm{R}$. The classification of stages of CKD is based on the value of GFR. The computation of eGFR is subject to error. This may give rise to misclassification of states. In this case we apply HMM instead of simple Markov model.

\section{Homogeneous continuous-time HMM}

Under the HMM, we assume that the unobserved true states follow Markov process with transition matrix $Q$ and the observed states are generated from the true underlying states through misclassification matrix. The effects of covariates on rates of transition and misclassification probabilities can be modeled using generalized regressions. A general model for the progression of disease and misclassification error can be explained as follows. Let $\mathrm{S}_{i t}^{c} \in[1, \ldots, 5]$ denotes the observed state generated by the hidden state $S_{i t}$. The observations $\mathrm{S}_{i t}^{c}$ are assumed to be conditionally independent of true states.

The observed states of the $i^{\text {th }}$ patient are denoted by $S_{i}^{c}=\left[\mathrm{S}_{i 1}^{c}, \ldots, \mathrm{S}_{i T_{i}}^{c}\right]$. The likelihood function for the observed categorical variable is given by

$$
f\left(S_{i}^{c}\right)=f\left(\mathrm{~S}_{i 1}^{c}, \ldots, \mathrm{S}_{i T_{i}}^{c}\right)
$$

$$
\begin{aligned}
& =\sum_{S_{i}} f\left(\mathrm{~S}_{i 1}^{c}, \ldots, \mathrm{S}_{i T_{i}}^{c} \mid S_{i 1}, \ldots \ldots . S_{i T_{i}}\right) f\left(S_{i 1}, \ldots \ldots S_{i T_{i}}\right) \\
& =\sum_{S_{i}} \prod_{t_{i}=t_{1}}^{T_{i}} f\left(S_{i t_{i}}^{c} \mid S_{\left.i t_{i}\right)}\right)\left\{f\left(S_{i 1}\right) \prod_{t_{i}=t_{2}}^{T_{i}} f\left(S_{i t_{i}} \mid S_{i t_{i}-1}\right)\right\}
\end{aligned}
$$

Where the summation is over all feasible paths of

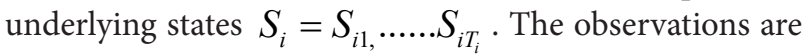
assumed to be independent given the series of true states. These are governed by misclassification probability matrix $\mathrm{E}$ and are independent of time. There is an assumption that disease stages are misclassified into the adjacent stage only. The misclassification probability matrix E for CKD is given by

$$
E=\left[\begin{array}{lllll}
e_{11} & e_{12} & 0 & 0 & 0 \\
e_{21} & e_{22} & e_{23} & 0 & 0 \\
0 & e_{32} & e_{33} & e_{34} & 0 \\
0 & 0 & e_{43} & e_{44} & 0 \\
0 & 0 & 0 & 0 & 0
\end{array}\right]
$$

Where $e_{r s}$ denotes the probability that the observed state is $s$ given that true state is $r$. The diagonal elements $e_{r r}$ of the misclassification matrix E indicate the correct classification of patients in the true stages of diseases. Rows of the matrix indicate the true states and columns of the matrix represent the observed states. Some entries of the matrix $\mathrm{E}$ have value zero where misclassification is not permitted. Presence of covariates affects the misclassification probabilities $e_{r s}$. Covariate effects on these probabilities can be estimated using multinomial logistic regression model (15) with baseline state $S_{0}$.

$\log \left(\frac{e_{r s}(t)}{e_{r s_{0}}(t)}\right)=\beta_{r s}^{\prime} \mathrm{Z}$

Where, $\beta_{r s}$ are regression coefficients and $Z$ are covariates.

The msm package in $\mathrm{R}$ has been used for estimating parameters of the model using maximum marginal likelihood method with numerical computations. There is a provision of fitting multistate Markov models in continuous time with or without misclassification error in msm package of software R. From the fitted model, the misclassification matrix, odds ratios for misclassification, observed and expected prevalence can be obtained using appropriate functions. Viterbi algorithm is the widely used method for reconstructing the most likely pathway or true pathway taken by a patient to reach the current stage without having the misclassification of stages. We can recreate the true stages actually experienced by a CKD patient to reach his current state. It was first suggested 
by Vitrebi et al (16) and later on Durbin (17), while Macdonald et al (18) described it for discrete time hidden Markov chains.

\section{Results}

The transition states of CKD patients in their subsequent visits have been summarized in Table 1. Generally, number of visits depends on the severity of the disease and awareness about the disease. Visits in stage 4 of CKD patients will have a higher frequency as compared to visits in lower stages. The table has been prepared by counting the number of transitions for each patient in their subsequent visits. The total number of times patients of stage 1 remains in stage 1 in their subsequent visits is 75 . The number of transitions to stage 5 from stage 1 , stage 2 , stage 3 and stage 4 are respectively 0,1 , 2 and 51. The number of transitions from higher stages to lower stages is zero as CKD is irreversible. Estimated transition intensities of simple homogeneous continuoustime multistate model based on Markov process have been shown in Table 2. The lower diagonal entries are zero as reverse transition are not allowed in CKD. Mean sojourn time of each state with $95 \%$ confidence interval and standard error are summarized in Table 3.

Estimated survival probability curves for stage 1, stage 2 , stage 3 and stage 4 have been shown in Figure 2. It is clear from the figure that there is a sharp decline in survivability of stage 4 patients nearing 1 year.

The estimated transition intensities for HMM are summarized in Table 4. Transition intensity corresponding to transition from stage 1 to stage 2 is 0.0405 whereas the transition intensity for transition from stage 4 to stage 5 is 0.346 . The mean sojourn time for each state for HMM have been summarized in Table 5. The table also shows

Table 1. Number of state transitions

\begin{tabular}{llllll}
\hline & Stage 1 & Stage 2 & Stage 3 & Stage 4 & Stage 5 \\
\hline Stage 1 & 75 & 8 & 4 & 3 & 0 \\
Stage 2 & 0 & 116 & 6 & 3 & 1 \\
Stage 3 & 0 & 0 & 293 & 29 & 2 \\
Stage 4 & 0 & 0 & 0 & 145 & 51 \\
\hline
\end{tabular}

the standard error and 95\% confidence interval for mean sojourn time for each stage of CKD. The mean sojourn time for stage 1 is approximately 16 years whereas it is about 2.9 years for CKD patient with disease stage as 4 .

Misclassification probabilities 1 for each state of CKD under HMM have been shown in Table 6. A CKD patient with stage 1 of disease will be misclassified as a patient of stage 2 with probability 0.211 . A patient of stage 2 will be misclassified as stage 1 patient with probability 0.280 . Table 7 shows the odds ratios for misclassification probabilities in the presence of categorical covariates.

Table 2. Estimated transition intensities

\begin{tabular}{llllll}
\hline & Stage 1 & Stage 2 & Stage 3 & Stage 4 & Stage 5 \\
\hline Stage 1 & -0.098 & 0.0599 & 0.0266 & 0.0115 & 0 \\
Stage 2 & 0 & -0.119 & 0.0725 & 0.0467 & 0 \\
Stage 3 & 0 & 0 & -0.115 & 0.115 & 0 \\
Stage 4 & 0 & 0 & 0 & -0.564 & 0.564 \\
\hline
\end{tabular}

Table 3. Mean sojourn times at different stages

\begin{tabular}{lccc}
\hline & Estimates & SE & Cl \\
\hline Stage 1 & 10.2030 & 2.6395 & $(6.1450,16.9407)$ \\
Stage 2 & 8.3829 & 2.6501 & $(4.5112,15.5772)$ \\
Stage 3 & 8.7051 & 1.5645 & $(6.1206,12.3808)$ \\
Stage 4 & 1.7734 & 0.2456 & $(1.3517,2.3265)$ \\
\hline
\end{tabular}

Table 4. Estimated transition intensities for misclassification model

\begin{tabular}{llllll}
\hline & Stage 1 & Stage 2 & Stage 3 & Stage 4 & Stage 5 \\
\hline Stage 1 & -0.0628 & 0.0405 & 0.0125 & 0.0098 & 0 \\
Stage 2 & 0 & -0.0835 & 0.0612 & 0.0223 & 0 \\
Stage 3 & 0 & 0 & -0.126 & 0.126 & 0 \\
Stage 4 & 0 & 0 & 0 & -0.346 & 0.346 \\
\hline
\end{tabular}

Table 5. Mean sojourn times for misclassification model

\begin{tabular}{lccc}
\hline & Estimates & SE & Cl \\
\hline Stage 1 & 15.923 & 3.5244 & $(5.438125,16.11562)$ \\
Stage 2 & 11.976 & 1.7256 & $(3.65214,14.5432)$ \\
Stage 3 & 7.936 & 1.8475 & $(5.231425,11.2814)$ \\
Stage 4 & 2.890 & 0.5413 & $(1.35174,2.926497)$ \\
\hline
\end{tabular}

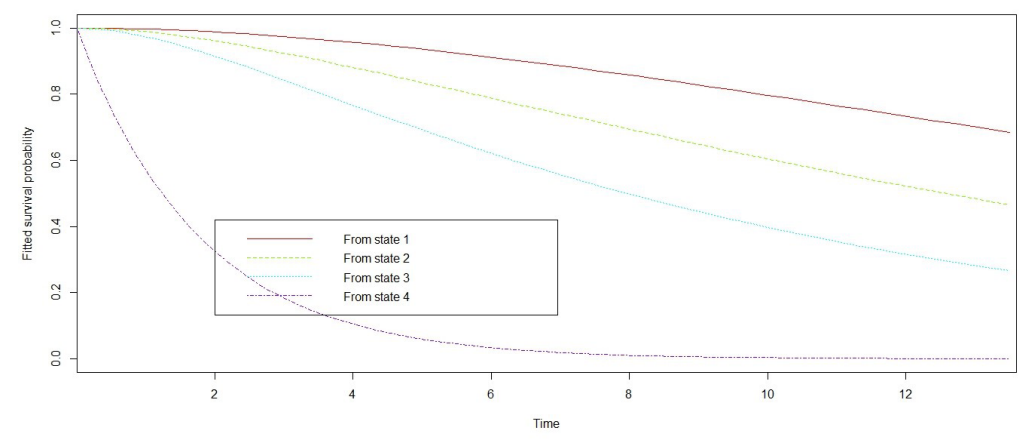

Figure 2. Plot of fitted survival probability. 
The probability of misclassification corresponding to the observed stage 2 given the true stage 1 for females is approximately 3.8 times more than that of males. Similarly, the misclassification probability for CKD patient corresponding to the observed stage 2 given the true stage 1 having hypertension is 2.28 times more than CKD patient having no hypertension and it is 3.56 times more for CKD patients with diabetes.

The odds ratios of misclassification probabilities in the presence of continuous type covariates have been summarized in Table 8 . For one year increase in the age of CKD patient, the odds are 3.52 times higher for the probability of misclassification corresponding to observed state 2 given true state 1 . Similarly for a CKD patient having 1 more unit of serum creatinine the probability of misclassification corresponding to observed state 2 given true state 1 is 2.34 times more.

The most likely state of randomly chosen CKD patients of stage 2 and stage 3 based on Viterbi algorithm have been summarized in Table 9. It is clear from the table that state of CKD patient number 27 has been misclassified at time 3.91. His actual stage is 3 but has been observed as stage 2 .

\section{Discussion}

$\mathrm{CKD}$ is a severe disease. The overall prevalence of CKD in India is about $17.2 \%$ with stage 1 , stage 2 , stage 3 , stage 4 and stage 5 as $7 \%, 4.3 \%, 4.3 \%$ and $0.8 \%$ and $0.8 \%$ respectively (19). Multistate Markov model is often used to describe the path of the progression of stage based

Table 6. Estimated misclassification probabilities

\begin{tabular}{lcc}
\hline Observed state & Actual state & Probabilities \\
\hline 1 & 1 & 0.789 \\
2 & 1 & 0.211 \\
1 & 2 & 0.280 \\
2 & 2 & 0.605 \\
3 & 2 & 0.115 \\
2 & 3 & 0.019 \\
3 & 3 & 0.901 \\
4 & 3 & 0.080 \\
3 & 4 & 0.027 \\
4 & 4 & 0.973 \\
\hline
\end{tabular}

Table 7. Odds ratios for misclassification probabilities under categorical variable

\begin{tabular}{llll}
\hline Misclassification & Sex & HTN & Diabetes \\
\hline e11 & & & \\
e12 & 3.782 & 2.281 & 3.563 \\
e21 & 0.003 & 0.627 & 0.443 \\
e22 & & & \\
e23 & 5.651 & 4.615 & 4.713 \\
e32 & 0.625 & 0.040 & 0.521 \\
e33 & & & \\
e34 & 0.753 & 4.611 & 2.590 \\
e43 & 0.075 & 0.004 & 0.524 \\
e44 & & & \\
\hline
\end{tabular}

disease. There is a possibility of misclassification of stages as measurements and clinical observations are prone to errors. HMM is more appropriate for such cases as it takes into account the probabilities of misclassification of stages as well. The number of transitions from state $i$ to state $i$ in their subsequent visits are more than moving to next higher stage. This shows the slow progression of the disease. The number of transitions to next higher stage is maximum for stage 4 indicating the rapid progression of disease in advance stage 4 . The diagonal entries of the transition matrix are negative which indicates the negation to the instantaneous risk of moving to other stage. The mean sojourn time for stage 1 is 15.923 years. It means that a patient of stage 1 spends on an average 15.923 years in stage 1 only before moving to stage 2 . The mean sojourn time for stage 4 is 2.890 years. This reveals the fact that the progression of disease is very slow in early stages as compared to higher stages. Misclassification of stages is possible for adjacent stages only. The probabilities of misclassification of stages are higher for early stages compared to advanced stages. A patient of CKD with stage 2 of disease is most likely to be misclassified as stage 1 of CKD patient if he is not suffering from diabetes and hypertension. On the other hand, there is a high probability that a patient of CKD suffering from diabetes

Table 8. Odds ratios for misclassification probabilities under continuous variables

\begin{tabular}{lcccccc}
\hline Missclassification & Age & BMI & Hb & Urea & Cr & Alb \\
\hline e11 & & & & & & \\
e12 & 3.521 & 4.171 & 0.003 & 0.0521 & 2.347 & 0.124 \\
e21 & 0.020 & 0.657 & 5.612 & 0.725 & 0.635 & 0.424 \\
e22 & & & & & & \\
e23 & 5.512 & 0.628 & 0.075 & 2.457 & 0.746 & 0.796 \\
e32 & 0.521 & 0.041 & 2.427 & 0.041 & 0.104 & 5.412 \\
e33 & & & & & & \\
e34 & 0.745 & 3.417 & 0.616 & 4.546 & 4.523 & 0.050 \\
e43 & 0.085 & 0.061 & 3.571 & 0.628 & 0.003 & 3.610 \\
e44 & & & & & & \\
\hline
\end{tabular}

Table 9. Viterbi sequence

\begin{tabular}{lccc}
\hline Subject & Time $(\mathbf{y})$ & Observed & Fitted \\
\hline 27 & 0 & 2 & 2 \\
27 & 0.375 & 2 & 2 \\
27 & 0.791667 & 2 & 2 \\
27 & 1.375 & 2 & 2 \\
27 & 1.666667 & 2 & 2 \\
27 & 3.083333 & 2 & 2 \\
27 & 3.916667 & 2 & 3 \\
27 & 4.25 & 4 & 4 \\
38 & 0 & 3 & 3 \\
38 & 0.916667 & 3 & 3 \\
38 & 3.583333 & 3 & 4 \\
38 & 5.833333 & 4 & 4 \\
38 & 6.916667 & 4 & 4 \\
38 & 7.833333 & 5 & 5 \\
\hline
\end{tabular}


and hypertension will be misclassified as stage 3 patient given that his true stage is stage 2. Early detection of disease helps in its treatment and delaying its progression to severe stages. The knowledge of natural history of disease progression is necessary for devising a policy by policymakers. It helps in reducing the economic burden of the treatment.

\section{Conclusion}

The HMM helps us in finding the natural history of the progression of CKD. The study concludes that the misclassification of stages in case of CKD may occur in the presence or absence of prognostic factors. Presence of covariates like hypertension and diabetes may overestimate the stages of CKD whereas their absence leads to underestimation of stages. Misclassification of stages may also take place due to error in computing GFR. The paper also concludes that the chances of misclassification of stages are more in the early stages of disease than advance stages of disease. There is an increase in mean sojourn time when misclassification of stages is taken into account.

\section{Limitations of the study}

This data set pertains to the patients from Delhi and adjoining areas. The people are more aware and medical facilities are good in this region. Baseline values of the prognostic factors have been used in the model for evaluating their impacts.

\section{Authors' contribution}

The idea/concept was given by the first author. All the authors have equally contributed to designing, analysis and preparation of the manuscript.

\section{Conflicts of interest}

The authors declare no conflict of interest.

\section{Ethical considerations}

Ethical issues (including plagiarism, data fabrication, double publication) have been completely observed by the authors.

\section{Funding/Support}

No agency or organization has funded this research work.

\section{References}

1. Tsai WC, Wu HY, Peng YS, Ko MJ, Wu MS, Hung KY, et al. Risk factors for development and progression of chronic kidney disease: a systematic review and exploratory metaanalysis. Medicine (Baltimore). 2016r;95:e3013. doi: 10.1097/MD.0000000000003013.
2. Varma PP. Prevalence of chronic kidney disease in IndiaWhere are we heading? Indian J Nephrol. 2015;25:133-5.

3. Agarwal SK, Srivastava RK. Chronic kidney disease in India: challenges and solutions. Nephron Clin Pract. 2009;111:c197-203. doi: 10.1159/000199460.

4. Grover G, Makhija N. On the Estimation of Survival and Death Probabilities under Myocardial Infarctions in the Presence of Competing Risks Based on an Illness-Death Model. J Commun Dis. 2010;42:1-17.

5. Grover G., Gadpayle AK, Swain PK, Deka B. A multistate Markov model based on CD4 cell count for HIV/AIDS patients on antiretroviral therapy (ART). Int J Stat Med Res. 2013;2:144-151.

6. Rabiner LR. A tutorial on hidden Markov models and selected applications in speech recognition. Proc IEEE. 1989;77:257-286. doi: 10.1109/5.18626.

7. Chen HH, Duffy SW, Tabar L. A Markov chain method to estimate the tumor progression rate from preclinical to clinical phase, sensitivity and positive predictive value for mammography in breast cancer screening. Statistician. 1996;45:307-17. doi: 10.1111/j.0006-341X.2001.00389.x.

8. Kirby AJ, Spiegelhalter DJ. Statistical modelling for the precursors of cervical cancer. In: Lange N, Ryan L, Billard L, Brillinger D, Conquest L, Greenhouse J, eds. Case Studies in Biometry. New York: Wiley; 1994.

9. Satten GA, Longini Jr IM. Markov chains with measurement error: estimating the 'true' course of a marker of the progression of human immunodeficiency virus disease. Appl Statist.1996;45:275-309. doi: 10.1214/15-AOAS810.

10. Guihenneuc-Jouyaux C, Richardson S, Longini IM Jr. Modelling markers of disease progression by a hidden Markov process: application to characterising CD4 cell decline. Biometrics. 2000;56:733-41.

11. Jackson $\mathrm{CH}$, Sharples LD. Hidden Markov models for the onset and progression of bronchiolitis obliterans syndrome in lung transplant recipients. Stat Med. 2002;21:113-28.

12. Grüger J, Kay R, Schumacher M. The validity of inferences based on incomplete observations in disease state models. Biometrics. 1991;47:595-605.

13. Cox DR, Miller HD. The Theory of Stochastic Processes. London: Chapman and Hall; 1965.

14. Marshall G, Jones RH. Multi-state Markov models and diabetic retinopathy. Stat Med. 1995;14:1975-83. doi:10.1002/sim.4780141804.

15. Jackson C. Multi-state modelling with R: the msm package. Version 1.6.5; 2017.

16. Viterbi A. Error bounds for convolutional codes and an asymptotically optimum decoding algorithm. IEEE Trans Inform Theory. 1967; 13:260-269, doi: 10.1109/ TIT.1967.1054010.

17. Durbin R, Eddy SR, Krogh A, Mitchison G. Biological sequence analysis: probabilistic models of proteins and nucleic acids. Cambridge university press, 1998.

18. MacDonald IL, Zucchini W. Hidden Markov and Other Models for Discrete-Valued Time Series, New York: Chapman and Hall, 1997.

19. Varma PP. Prevalence of chronic kidney disease in IndiaWhere are we heading? Indian J Nephrol. 2015;25:133-5.

Copyright $\odot 2019$ The Author(s); Published by Society of Diabetic Nephropathy Prevention. This is an open-access article distributed under the terms of the Creative Commons Attribution License (http://creativecommons.org/licenses/by/4.0), which permits unrestricted use, distribution, and reproduction in any medium, provided the original work is properly cited. 